\title{
Minimal flavour violation extensions of the seesaw
}

\author{
Rodrigo Alonso, ${ }^{a, b}$ Gino Isidori, ${ }^{c}$ Luca Merlo, ${ }^{d, e}$ Luis Alfredo Muñoz ${ }^{f, g}$ and \\ Enrico Nardi ${ }^{a, b, c}$ \\ ${ }^{a}$ Departamento de Física Teórica, C-XI, Facultad de Ciencias, Universidad Autónoma de Madrid, \\ C.U. Cantoblanco, 28049 Madrid, Spain \\ ${ }^{b}$ Instituto de Física Teórica, IFT-UAM/CSIC, \\ Nicolas Cabrera 15, C.U. Cantoblanco, 28049 Madrid, Spain \\ ${ }^{c}$ INFN, Laboratori Nazionali di Frascati, \\ Via Enrico Fermi 40, I-00044 Frascati, Italy \\ ${ }^{d}$ Physik-Department, Technische Universität München, \\ James-Franck-Strasse, D-85748 Garching, Germany \\ ${ }^{e}$ TUM Institute for Advanced Study, Technische Universität München, \\ Lichtenbergstrasse 2a, D-85748 Garching, Germany \\ ${ }^{f}$ Instituto de Física, Universidad de Antioquia, \\ A.A. 1226 Medellín, Colombia \\ ${ }^{g}$ Instituto Tecnólogico Metropolitano, \\ Calle 73 No. 76A-354 Medellín, Colombia \\ E-mail: rodrigo.alonso@uam.es, gino.isidori@lnf.infn.it, \\ luca.merlo@ph.tum.de, lmunoz@fisica.udea.edu.co, \\ enrico.nardi@lnf.infn.it
}

ABSTRACT: We analyze the most natural formulations of the minimal lepton flavour violation hypothesis compatible with a type-I seesaw structure with three heavy singlet neutrinos $N$, and satisfying the requirement of being predictive, in the sense that all LFV effects can be expressed in terms of low energy observables. We find a new interesting realization based on the flavour group $\mathrm{SU}(3)_{e} \times \mathrm{SU}(3)_{\ell+N}$ (being $e$ and $\ell$ respectively the $\mathrm{SU}(2)$ singlet and doublet leptons). An intriguing feature of this realization is that, in the normal hierarchy scenario for neutrino masses, it allows for sizeable enhancements of $\mu \rightarrow e$ transitions with respect to LFV processes involving the $\tau$ lepton. We also discuss how the symmetries of the type-I seesaw allow for a strong suppression of the $N$ mass scale with respect to the scale of lepton number breaking, without implying a similar suppression for possible mechanisms of $N$ production.

Keywords: Neutrino Physics, Global Symmetries, Rare Decays

ArXiv EPRINT: 1103.5461 


\section{Contents}

1 Introduction 1

2 Minimal effective theories for the seesaw 2

2.1 Breaking of the U(1) symmetries and size of the LFV effects 4

$\begin{array}{lll}2.2 & \text { Two predictive cases } & 6\end{array}$

2.3 Phenomenology 8

$\begin{array}{lll}3 & \text { Conclusions } & 11\end{array}$

\section{Introduction}

The Minimal Flavour Violation (MFV) hypothesis [1-4] is, in short, the assumption that the sources of flavour symmetry breaking present in the SM Lagrangian determine completely the structure of flavour symmetry breaking also beyond the SM. In the quark sector there is a unique way to implement this hypothesis: the two quark SM Yukawa couplings are identified as the only relevant breaking terms of the $\mathrm{SU}(3)^{3}$ quark-flavour symmetry [4]. In contrast, in the lepton sector there is no unique way to realize MFV. The SM by itself cannot accommodate Lepton Flavour Violation (LFV): since there is a single set of Yukawa couplings (the ones for the charged leptons), they can always be brought in diagonal form by rotating the three $\mathrm{SU}(2)_{L}$-doublets $\left(\ell_{\alpha}\right)$ and the three right-handed $(\mathrm{RH}) \mathrm{SU}(2)_{L}$-singlets $e_{\alpha}(\alpha=e, \mu, \tau)$. However, LFV is observed in neutrino oscillation experiments. It is then interesting to formulate an extension of the MFV hypothesis to the lepton sector, or a Minimal Lepton Flavour Violation (MLFV) hypothesis, whose starting point is not the SM Lagrangian but an effective Lagrangian able to describe also the observed LFV effects in the neutrino sector. The problem is that we do not know which physics beyond the SM is responsible for the observed neutrino masses and LFV effects, and different extensions of the SM correspond to different formulations of the MLFV hypothesis.

The simplest way to extend the SM to include (strongly suppressed) neutrino masses is by adding to the SM Lagrangian the dimension-five Weinberg operator [5]:

$$
\begin{aligned}
\mathcal{L}_{\text {eff }}^{\left(m_{\nu}\right)} & =\mathcal{L}_{Y}^{\mathrm{SM}}+\mathcal{L}_{D 5}, \\
\mathcal{L}_{Y}^{\mathrm{SM}} & =-\bar{\ell}_{\alpha} Y^{\alpha \beta} e_{\beta} H+\text { h.c. } \\
\mathcal{L}_{D 5} & =-\frac{g_{\alpha \beta}}{2 M}\left(\bar{\ell}_{\alpha}^{c} \tilde{H}\right)\left(\tilde{H}^{T} \ell_{\beta}\right)+\text { h.c. },
\end{aligned}
$$

where $H$ is the Higgs field, ${ }^{1}$ and $M$ is a high scale related to the breaking of the lepton number $(L)$. Although the appearance of neutrino masses and LFV is linked to the introduction of a non-renormalizable operator, the MLFV formulation based on eq. (1.1) has a

\footnotetext{
${ }^{1}$ Here and in the following the indexes on the lepton fields refer only to the flavour structure; $\tilde{H}=i \tau_{2} H^{*}$, $\ell^{c}=C \gamma^{0} \ell^{*}$, and the appropriate $\mathrm{SU}(2)_{L}$ index contraction with the lepton doublets $\ell$ is left understood.
} 
minimal field content and is minimal also in terms of the relevant flavour symmetry. The latter can be chosen to be $\mathrm{U}(3)_{\ell} \times \mathrm{U}(3)_{e}$ : the largest symmetry group of the gauge invariant kinetic terms of the SM leptons. Factorizing the two U(1) groups identified by lepton number and hypercharge, under the remaining semi-simple flavour subgroup $\mathrm{SU}(3)_{\ell} \times \mathrm{SU}(3)_{e}$ the leptons transform as $\ell \sim(3,1)$ and $e \sim(1,3)$. Formal invariance of the effective Lagrangian under $\mathrm{SU}(3)_{\ell} \times \mathrm{SU}(3)_{e}$ is then recovered by promoting the couplings $Y_{\alpha \beta}$ and $g_{\alpha \beta}$ to spurion fields with the assignments $Y \sim(3, \overline{3})$ and $g \sim(\overline{6}, 1)$.

The LFV operators of dimension-six, that are naturally present in the effective theory approach, conserve $B-L[5]$ and are suppressed by a new effective scale $\Lambda$ not necessarily related to $M$ that, in this minimal scheme, is the $L$-breaking scale. The extremely tight limits on $B$ violating processes then imply that if $\Lambda \ll \Lambda_{G U T}$, then the dimension-six operators must conserve $B$, and thus $L$ as well. According to the MLFV ansatz, these operators are built only in terms of SM fields and spurions, preserving formal invariance under the flavour group. Besides the requirement of a sufficiently low scale $\Lambda$, the possibility of observing new LFV effects also requires rather large values of $g_{\alpha \beta}$. Since the magnitude of $g_{\alpha \beta} / M$ is fixed by the light neutrino mass scale, a large $g_{\alpha \beta}$ requires a correspondingly high scale $M$, and this results in a large hierarchy $\Lambda / M \ll 1$. A detailed study of this framework is given in $[6,7]$ and will not be repeated here.

The main drawback of the MLFV ansatz based on eq. (1.1) is that it cannot be linked to several dynamical models for neutrino masses based on the seesaw mechanism. This is why in [6], and later on also in [8,9], a second scenario, with an extended field content and a different flavour group has been analyzed. Alternative formulations of the MLFV hypothesis have also been proposed in [10-12]. Once the field content of the theory is extended, there are in principle several possibilities to define the flavour symmetry and a consistent minimal set of spurions. However, if we restrict the attention to the popular type-I seesaw models [13-16], the choice is restricted: the purpose of this paper is to analyze the most natural formulations of the MLFV hypothesis compatible with a type-I seesaw structure with three singlet Majorana neutrinos. We find a new MLFV realization that allows for sizable enhancements of the LFV violating processes involving the lighter generations, and thus is phenomenologically interesting.

\section{Minimal effective theories for the seesaw}

In order to define a MLFV effective theory in the context of a type-I seesaw structure, we assume that in addition to the SM leptons $(\ell$ and $e$ ) at high energies there is at least another set of dynamical fields carrying lepton flavour: the three SM singlets heavy Majorana neutrinos $N$. The largest group of flavour transformations commuting with the gauge invariant kinetic terms of the lepton fields $\ell, N$ and $e$ is $\mathcal{G}=\mathrm{U}(3)_{\ell} \times \mathrm{U}(3)_{N} \times \mathrm{U}(3)_{e}$. We assume that $\mathcal{G}$, or some subgroup of $\mathcal{G}$, is the relevant group of flavour transformations, and that this symmetry is broken at some high scale $\Lambda_{F}$ larger than the scale of the RH neutrino masses. Most important, we require that all the relevant symmetry-breaking terms (the spurions) can be identified with the couplings appearing in the seesaw Lagrangian, namely the renormalizable mass terms of the three basic sets of lepton fields. 
Integrating out the heavy degrees of freedom, the effective Lagrangian relevant at energies below $\Lambda_{F}$ can be decomposed as

$$
\mathcal{L}_{\text {eff }}\left(E<\Lambda_{F}\right)=\mathcal{L}_{\text {kin }}(N, \ell, e)+\mathcal{L}_{\text {seesaw }}(N, \ell, e ; H)+\Delta \mathcal{L}_{\Lambda_{F}}
$$

where

$$
-\mathcal{L}_{\text {seesaw }}(N, l, e ; H)=\epsilon_{e} \bar{\ell}_{\alpha} Y_{e}^{\alpha \beta} e_{\beta} H+\epsilon_{\nu} \bar{\ell}_{\alpha} Y_{\nu}^{\alpha j} N_{j} \tilde{H}+\frac{1}{2} \epsilon_{\nu}^{2} \mu_{L} \bar{N}_{i}^{c} Y_{M}^{i j} N_{j}+\text { h.c.. }
$$

$\Delta \mathcal{L}_{\Lambda_{F}}$ in eq. (2.1) denotes higher-dimensional operators involving $N, \ell$, and $e$, suppressed by inverse powers of $\Lambda_{F}$, as well as other renormalizable and non-renormalizable interactions of these lepton fields with additional flavour-carrying degrees of freedom that are relevant below $\Lambda_{F}$. In order to analyze the transformation properties of the $\mathcal{G}$-breaking spurions appearing in $\mathcal{L}_{\text {seesaw }}$, we decompose the symmetry group as follows

$$
\mathcal{G}=\mathrm{U}(1)_{Y} \times \mathrm{U}(1)_{L} \times \mathrm{U}(1)_{R} \times \mathcal{G}_{F}, \quad \mathcal{G}_{F}=\mathrm{SU}(3)_{\ell} \times \mathrm{SU}(3)_{N} \times \mathrm{SU}(3)_{e},
$$

where $\mathrm{U}(1)_{Y}$ and $\mathrm{U}(1)_{L}$ correspond to hypercharge (that remains unbroken) and to total lepton number, respectively. The remaining Abelian factor can be identified either with $\mathrm{U}(1)_{e}$ or with $\mathrm{U}(1)_{N}$, corresponding respectively to global phase rotations of the $\mathrm{RH}$ charged leptons or RH neutrinos, and we will generically denote it by $\mathrm{U}(1)_{R}$. By construction, we assume that $Y_{e, \nu, M}$ are dimensionless spurions that carry no $\mathrm{U}(1)_{R}$ charges, and thus break only $\mathcal{G}_{F}$. The transformation properties of the lepton fields and of the spurions under $\mathcal{G}_{F}$ are

$$
\begin{aligned}
& \ell \sim(3,1,1) \quad N \sim(1,3,1) \quad e \sim(1,1,3), \\
& Y_{\nu} \sim(3, \overline{3}, 1) \quad Y_{M} \sim(1, \overline{6}, 1) \quad Y_{e} \sim(3,1, \overline{3}) .
\end{aligned}
$$

As regards the two broken Abelian factors, $\mathrm{U}(1)_{L}$ is broken (by two units) by $\mu_{L}$, that is a spurion with dimension of a mass, while $\mathrm{U}(1)_{R}$ is broken by a dimension-less spurion $\epsilon_{R}$, where $\epsilon_{R}$ denotes $\epsilon_{e}$ or $\epsilon_{\nu}$.

At this point we are ready to make one more step, integrating out from the effective Lagrangian eq. (2.1) the heavy RH neutrinos with masses of order $\epsilon_{\nu}^{2} \mu_{L} \ll \Lambda_{F}$. We also assume that at some different scale $\Lambda \ll \Lambda_{F}$, presumably around or somewhat above the electroweak scale, other states carrying flavour are present, and we integrate them out as well. The resulting effective Lagrangian can be decomposed as

$$
\mathcal{L}_{\text {eff }}(E<\Lambda)=\mathcal{L}_{\mathrm{SM}}+\mathcal{L}_{D 5}^{\text {seesaw }}+\frac{1}{\Lambda^{2}} \sum_{i} c_{i} O_{i}^{(6)}+\ldots,
$$

where $\mathcal{L}_{D 5}^{\text {seesaw }}$ is nothing but the Weinberg operator, whose coupling can now be determined in terms of the spurions appearing in eq. (2.2). The $O_{i}^{(6)}$ denote generic dimensions-six operators written in terms of the SM fields and of the spurions, and the dots denotes operators of higher dimension. As shown in [5], dimensions-six operators written in terms of the SM fields conserve $B-L$, and since we have not introduced (dangerous) sources of $B$ violation, then the operators $O_{i}^{(6)}$ must conserve separately $L$. This is the reason 
why the scale $\Lambda$ can be substantially lower than $\Lambda_{F}$ and of the $\mathrm{RH}$ neutrino mass scale. Note in particular that $\mathrm{U}(1)_{N}$ breaking and $\epsilon_{\nu}$ only affect the $\mathrm{RH}$ neutrino masses, without affecting in any way the Weinberg operator.

As far as the flavour structure of the $O_{i}^{(6)}$ is concerned, our assumptions about the breaking of $\mathcal{G}_{F}$ imply the following

I. All higher-dimensional operators must be formally invariant under $\mathcal{G}_{F}$ once the transformation properties of the fields eq. (2.4) and of the spurions eq. (2.5) are taken into account.

As is pointed out in [6], this condition alone is not sufficient to obtain an effective theory that is predictive, since the flavour structure of the three spurions $Y_{\nu}, Y_{M}$ and $Y_{e}$ cannot be determined from low-energy data alone. A predictive MLFV formulation must satisfy an additional working hypothesis:

II. The flavour structure of the spurions must be determined in terms of low energy observables, namely the PMNS mixing matrix and the light neutrino mass eigenvalues.

The only way this second hypothesis can be satisfied is by restricting the form of the spurions $Y_{i}$ in such a way that the relevant LFV combinations will depend on a reduced number of parameters. As we show in the following, this goal can be naturally obtained by assuming that the underlying flavour symmetry corresponds to a subgroup of $\mathcal{G}_{F}$ rather than to the full flavour group $\mathrm{SU}(3)^{3}$.

\subsection{Breaking of the U(1) symmetries and size of the LFV effects}

Before analyzing the possible subgroups of $\mathcal{G}_{F}$ leading to predictive frameworks, it is worth to discuss in general terms the overall size of the LFV effects and its connection to the breaking of $\mathrm{U}(1)_{R}$. The explicit structure of the Weinberg operator obtained by integratingout the RH neutrinos, and the corresponding light neutrino mass matrix, are

$$
\mathcal{L}_{D 5}^{\text {seesaw }}=\frac{1}{\mu_{L}}(\bar{\ell} \tilde{H}) Y_{\nu} \frac{1}{Y_{M}} Y_{\nu}^{T}\left(\tilde{H}^{T} \ell^{c}\right) \quad \longrightarrow \quad m_{\nu}^{\dagger}=\frac{v^{2}}{\mu_{L}} Y_{\nu} \frac{1}{Y_{M}} Y_{\nu}^{T}=U \mathbf{m}_{\nu} U^{T}
$$

where $v=\langle H\rangle$ is the Higgs vacuum expectation value (vev), $U$ is the PMNS matrix and $\mathbf{m}_{\nu}=\operatorname{diag}\left(m_{\nu_{1}}, m_{\nu_{2}}, m_{\nu_{3}}\right)$. Note that since the Weinberg operator does not break $\mathrm{U}(1)_{R}$, the overall size of $\mathbf{m}_{\nu}$ depends only on the lepton-number violating scale $\mu_{L}$, but not on $\epsilon_{e, \nu}$. Without loss of generality we can rotate $Y_{e}$ and $Y_{M}$ to a basis where they are both diagonal, and in terms of mass eigenvalues they can be written as:

$$
\begin{aligned}
Y_{e} & =\frac{1}{\epsilon_{e} v} \operatorname{diag}\left(m_{e}, m_{\mu}, m_{\tau}\right), \\
Y_{M} & =\frac{1}{\epsilon_{\nu}^{2} \mu_{L}} \operatorname{diag}\left(M_{1}, M_{2}, M_{3}\right) .
\end{aligned}
$$

These equations show that the overall size of $Y_{e}$ and $Y_{M}$ is controlled by the Abelian spurion, and the same is true for $Y_{\nu}$. A natural choice for the size of the $\mathrm{U}(1)_{R}$ breaking is the one 
that allows us to keep $\mathcal{O}(1)$ entries in the $Y_{i}$ matrices. In the case of the light-neutrino mass matrix, this choice unambiguously points to a very large $L$-breaking scale

$$
\mu_{L} \sim \frac{v^{2}}{\sqrt{\Delta m_{\mathrm{atm}}^{2}}} \approx 6 \times 10^{14} \mathrm{GeV}
$$

As far as $\epsilon_{e}$ and $\epsilon_{\nu}$ are concerned, we can envisage two possibilities, depending if the additional broken Abelian symmetry is $\mathrm{U}(1)_{e}$ or $\mathrm{U}(1)_{N}$. In the first case we can set $\epsilon_{e} \approx$ $m_{\tau} / v$, providing a natural explanation for the smallness of the charged-lepton Yukawa coupling, but then naturalness suggests $\epsilon_{\nu} \approx 1$, i.e. very heavy $\mathrm{RH}$ neutrinos with masses of $\mathcal{O}\left(\mu_{L}\right)$. In the second case $\epsilon_{e} \approx 1,{ }^{2}$ but we are free to assume $\epsilon_{\nu} \ll 1$ as would naturally result from an approximate $\mathrm{U}(1)_{N}$ symmetry. In this case the $\mathrm{RH}$ neutrinos could have masses well below the $L$-breaking scale, and possibly within the reach of future experiments [17]. From the phenomenological point of view this second one is clearly the most interesting choice, and is the one we will adopt from now on.

As we have already discussed, the dimension-six operators contributing to low-energy LFV processes are invariant under $\mathrm{U}(1)_{L}$ and, by construction, they are also invariant under $\mathrm{U}(1)_{N}$. As a result, assuming that the $Y_{i}$ spurions have $\mathcal{O}(1)$ entries implies that the overall scale of LFV effects is controlled only by the effective scale $\Lambda$. The generic structure of the most relevant LFV operators is

$$
\begin{aligned}
O_{L R}^{(6)} & =\bar{\ell}_{\alpha}\left(\Delta_{8} Y_{e}\right)^{\alpha \beta}(\sigma \cdot F) e_{\beta} H, \\
O_{L L 1}^{(6)} & =\bar{\ell}_{\alpha} \Gamma \Delta_{8}^{\alpha \beta} \ell_{\beta} \times \bar{f} \Gamma^{\prime} f \\
O_{L L 2}^{(6)} & =\bar{\ell}_{\alpha} \Gamma \Delta_{8}^{\alpha \beta} \ell_{\beta} \times \bar{\ell}_{\alpha} \Gamma^{\prime} \Delta_{8}^{\alpha \beta} \ell_{\beta}, \\
O_{L L 3}^{(6)} & =\bar{\ell}_{\alpha} \Gamma \Delta_{6}^{\alpha \beta} \ell^{c} \times \bar{\ell}_{\alpha}^{c} \Gamma^{\prime} \Delta_{\overline{6}}^{\alpha \beta} \ell_{\beta},
\end{aligned}
$$

where $\Delta_{8}, \Delta_{6}$, and $\Delta_{\overline{6}}$ are $\mathrm{SU}(3)_{e} \times \mathrm{SU}(3)_{N}$ singlets combinations of the $Y_{i}$ transforming as 8,6 and $\overline{6}$ of $\mathrm{SU}(3)_{\ell}$, respectively ( $\Gamma$ stands for generic Dirac structures and/or $\mathrm{SU}(2)_{L}$ matrices, $F$ generically denotes the field strength of $\mathrm{SU}(2)_{L} \times \mathrm{U}(1)_{Y}$ gauge fields, while $f$ stands for SM leptons or quarks). Because of the hierarchical structure of $Y_{e}$, LFV bilinears with two $\mathrm{RH}$ charged leptons (such as $\bar{e} Y_{e} \Delta_{8} Y_{e} e$ ) are always suppressed with respect to the corresponding LH terms in eqs. (2.12)-(2.14) and we neglect them.

Considering terms with up to two $Y_{\nu}$ and two $Y_{M}$, we can write the following contributions to the operators eqs. (2.11)-(2.14):

$$
\Delta_{8}^{(1)}=Y_{\nu} Y_{\nu}^{\dagger}, \quad \Delta_{6}^{(1)}=Y_{\nu} Y_{M}^{\dagger} Y_{\nu}^{T}, \quad \Delta_{8}^{(2)}=Y_{\nu} Y_{M}^{\dagger} Y_{M} Y_{\nu}^{\dagger},
$$

with $\Delta_{\overline{6}}^{(1)}=\left(\Delta_{6}^{(1)}\right)^{\dagger}$. In the mass diagonal basis of eqs. (2.8)-(2.9) all LFV effects are associated to $Y_{\nu}$, which is a generic complex $3 \times 3$ matrix corresponding to 15 physical parameters. In the absence of further assumptions we will not be able to determine all these parameters from eq. (2.7). However, as anticipated, predictive frameworks can be obtained by choosing as the underlying flavour symmetry some suitable subgroup of $\mathcal{G}_{F}$.

\footnotetext{
${ }^{2}$ If $\epsilon_{e} \approx 1$, the suppression of the charged-lepton masses could still be justified in a multi-Higgs scenario by the hierarchy in the vevs with different hypercharge: $v_{d} \ll v_{u}$.
} 


\subsection{Two predictive cases}

There are basically two natural criteria that we can follow to relate the LFV structures $\Delta$ to the observables in eq. (2.7). The two criteria, which can be formulated in terms of general symmetry hypotheses, allow us to assume that in a given basis either $Y_{\nu}$ or $Y_{M}$ corresponds to the identity matrix in flavour space $I_{3 \times 3}$.

A. $\mathrm{SU}(3)_{\ell} \times \mathrm{SU}(3)_{N} \rightarrow \mathrm{SU}(3)_{\ell+N}$.

If we assume that $\ell$ and $N$ belongs to the fundamental representation of the same $\mathrm{SU}(3)$ group, then in a generic basis $Y_{\nu}$ must be a unitary matrix (and thus it can be always rotated to the identity matrix by a suitable unitary transformation of the $\mathrm{RH}$ neutrinos). This condition is sufficient to allow inverting the seesaw formula in eq. (2.7). By doing so we find

$$
\begin{aligned}
& \Delta_{6}=\Delta_{6}^{\dagger}=\left[\left(Y_{\nu} \frac{1}{Y_{M}} Y_{\nu}^{T}\right)^{-1}\right]^{\dagger}=\frac{v^{2}}{\mu_{L}} U \frac{1}{\mathbf{m}_{\nu}} U^{T}, \\
& \Delta_{8}^{(2)}=\Delta_{6} \cdot \Delta_{6}^{\dagger}=\frac{v^{4}}{\mu_{L}^{2}} U \frac{1}{\mathbf{m}_{\nu}^{2}} U^{\dagger},
\end{aligned}
$$

while $\Delta_{8}^{(1)}=I_{3 \times 3}$ and gives no LFV effects.

The choice of a unitary $Y_{\nu}$ can also be justified on a different basis. According to a general theorem [18] if the $N$ 's belong to an irreducible 3-dimensional representation of a non-Abelian group, then $Y_{\nu}$ is (proportional to) a unitary matrix. Let us recall that models for neutrino masses based on discrete non-Abelian flavour symmetries have proved to be quite successful in reproducing the structure of the PMNS matrix. This is generally related to the fact that in first approximation the symmetry implies a tri-bimaximal (TBM) [19, 20] mixing pattern that is a good approximation to PMNS. We can then picture a situation where in a first step of the flavour symmetry breaking $\mathrm{SU}(3)_{N}$ breaks to a non-Abelian discrete subgroup having irreducible 3dimensional representations to which the $N$ 's are assigned. In this case $Y_{\nu}$ can be non-trivial but must be proportional to a unitary matrix (while $Y_{M}$ is clearly $\propto I$ or vanishing). In a second step, when the discrete symmetry is broken, $Y_{M}$ acquires a non-trivial structure, while corrections to $Y_{\nu}$ can be quantified to remain at the level of the deviations of $U$ from TBM, that is small. Several models based on discrete non-Abelian symmetry that yield a unitary $Y_{\nu}$ or $Y_{\nu} \propto I_{3 \times 3}$ have been constructed, and a long list of references, properly classified according to these two possibilities, can be found in [21].

A detailed phenomenological analysis of this scenario is presented in the next session. The main distinctive feature with respect to the case based on the $O(3)_{N}$ symmetry analyzed in [6] (see point B. below) is that, due to the inverse $\mathbf{m}_{\nu}$ dependence in eqs. (2.16)-(2.17), LFV processes are enhanced when the lighter neutrinos mass eigenvalues are involved. This implies, in particular, a potentially strong enhancement of $\mu \rightarrow e \gamma$ in the normal-hierarchy (NH) case. 
Because of the nine conditions on the matrix $Y_{\nu}$ implied by unitarity, the eighteen independent parameters of the general type I seesaw model are reduced to nine. The free parameters are easily red out from the second relation in eq. (2.7), from which we have:

$$
Y_{\nu}=U, \quad m_{\nu_{i}}=\epsilon_{\nu}^{2} \frac{v^{2}}{M_{i}}
$$

where $M_{i}=\epsilon_{\nu}^{2} \mu_{L} Y_{M_{i}}$ are the heavy neutrino masses. Thus, while the PMNS matrix is not constrained, and corresponds as usual to three real angles plus three complex phases, the light neutrino masses are inversely proportional to the heavy masses, giving only three additional parameters. Other phenomenologically interesting features of this scenario, that are largely independent of the particular pattern of flavour symmetry reduction $\mathrm{SU}(3)_{\ell} \times \mathrm{SU}(3)_{N} \rightarrow \mathrm{SU}(3)_{\ell+N}$ but are mainly related to the breaking of $\mathrm{U}(1)_{N}$ and to assumptions about the size of the spurion $\epsilon_{N}$, are:

- Being unrelated to the breaking of $\mathrm{U}(1)_{L}$ and $\mathrm{U}(1)_{N}$, the LFV violating scale $\Lambda$ can be as low as permitted by present phenomenological constraints.

- Assuming $\mathcal{O}(1)$ entries for the non-Abelian spurions $Y_{i}$, double LFV processes (such as $\tau \rightarrow e e \bar{\mu}$ ) are not necessarily strongly suppressed with respect to single LFV process (such as $\tau \rightarrow e \bar{e} \mu$ ).

- As long as $\epsilon_{\nu} \gg v / \mu_{L}$ the light neutrino masses do not depend on its value. In contrast, the masses of the $\mathrm{RH}$ neutrinos are suppressed with respect to $\mu_{L}$ by two powers of $\epsilon_{\nu}$. Then, even if the $L$-number breaking scale $\mu_{L}$ is generically large eq. (2.10), if the $\mathrm{U}(1)_{N}$ breaking is small $\left(\epsilon_{\nu} \lesssim 10^{-5}\right) N$ states with masses of a few $\mathrm{TeV}$ (or even lower) are an open possibility.

- The symmetries of this scenario imply that $\mathrm{U}(1)_{N}$ conserving operators of the form $(\bar{N} N) \cdot(\bar{q} q)$ (where $q$ denote quark fields) are not suppressed by any power of $\epsilon_{\nu}$. Therefore, in the absence of other suppressing effects, we can even envisage the possibility that the $N$ 's can be produced at colliders.

B. $\mathrm{SU}(3)_{N} \rightarrow O(3)_{N} \times C P$.

Assuming that the flavour group acting on the RH neutrinos is $O(3)_{N}$ rather than $\mathrm{SU}(3)_{N}$, implies that $Y_{M}$ must be proportional to $I_{3 \times 3}$. However, this condition alone is not enough to deduce the structure of $Y_{\nu}$ from the seesaw formula: this requirement, (and hence the predictivity of the theory) is fulfilled only if we further assume that $Y_{\nu}$ is real $Y_{\nu}^{\dagger}=Y_{\nu}^{T}$ (which follows from imposing CP invariance) [6]. In this case, since the Majorana mass term has a trivial structure, all LFV effects stem from the (real) Yukawa coupling matrices:

$$
\Delta_{6}=\Delta_{8}^{(1)}=\Delta_{8}^{(2)}=Y_{\nu} Y_{\nu}^{T}=\frac{\mu_{L}}{v^{2}} U \mathbf{m}_{\nu} U^{T} .
$$

The implications for LFV processes of this scenario have been analyzed in [6] and we refer to this paper for further details. 


\subsection{Phenomenology}

In this section we discuss the dependence of LFV processes, and in particular of the radiative decay $\ell_{i} \rightarrow \ell_{j} \gamma$, on $\Delta_{8}^{(2)}$ defined in eq. (2.17). In order to compare the relevance of different decay channels we define the normalized branching fractions [6]:

$$
B_{\ell_{i} \rightarrow \ell_{j} \gamma} \equiv \frac{\Gamma\left(\ell_{i} \rightarrow \ell_{j} \gamma\right)}{\Gamma\left(\ell_{i} \rightarrow \ell_{j} \nu_{i} \bar{\nu}_{j}\right)}
$$

We are interested in studying quantitatively ratios of these quantities for different types of radiative decays. These ratios simply reduce to ratios of the modulus squared of the corresponding $\Delta_{8}^{(2)}$ entries:

$$
\frac{B_{\ell_{i} \rightarrow \ell_{j} \gamma}}{B_{\ell_{k} \rightarrow \ell_{m} \gamma}}=\frac{\left|\left(\Delta_{8}^{(2)}\right)_{i j}\right|^{2}}{\left|\left(\Delta_{8}^{(2)}\right)_{k m}\right|^{2}},
$$

Omitting the prefactor $\frac{v^{4}}{\mu_{L}^{2}}$ that cancels in the ratios, the relevant LFV structures then reduce to $\Delta_{8}^{(2)} \rightarrow U \frac{1}{\mathbf{m}_{\nu}^{2}} U^{\dagger}$. We generate random values for these quantities allowing the neutrino parameters to vary within their (approximate) $2 \sigma$ c.l. experimental intervals $[22-25]$ :

$$
\begin{aligned}
\Delta m_{\mathrm{sol}}^{2}: & (7.3-8.0) \times 10^{-5} \mathrm{eV}^{2}, \\
\Delta m_{\mathrm{atm}}^{2}: & (2.2-2.6) \times 10^{-3} \mathrm{eV}^{2}, \\
\sin ^{2} \theta_{12}: & 0.28-0.35 \\
\sin ^{2} \theta_{23}: & 0.35-0.61 \\
\sin ^{2} \theta_{13}: & 0.0-0.04
\end{aligned}
$$

For the $\mathrm{NH}$ and inverted hierarchy $(\mathrm{IH})$ we restrict the range of variation of the lightest mass eigenvalue respectively to $m_{\nu_{1}}\left(m_{\nu_{3}}\right) \leq 0.1 \mathrm{eV}$, while the CP phase $\delta$, that enters all the formulas through $\cos \delta$, is varied in the interval $[0-\pi]$ (the Majorana phases are of course irrelevant for $\Delta L=0$ processes).

In figure 1 we present the results for the ratios $\frac{B_{\mu \rightarrow e \gamma}}{B_{\tau \rightarrow \mu \gamma}}$ (left panel) and $\frac{B_{\mu \rightarrow e \gamma}}{B_{\tau \rightarrow e \gamma}}$ (right panel) as a function of the lightest mass eigenvalue $m_{\nu_{l}}=m_{\nu_{1}}$ (NH: green points) and $m_{\nu_{l}}=m_{\nu_{3}}$ (IH: red points) while all the other parameters are varied aleatorily in the given intervals. Note that in this figure, as well as in all the other figures below, the density of points depends on arbitrary details of the sampling procedure, and should not be interpreted as related to the the likelihood of regions differently populated.

From the first panel we see that for NH and small values of $m_{\nu_{1}} \lesssim 10^{-2} \mathrm{eV}$ we generically have $B_{\mu \rightarrow e \gamma}>B_{\tau \rightarrow \mu \gamma}$. The enhancement of $B_{\mu \rightarrow e \gamma}$ is obviously due to $\mathbf{m}_{\nu}^{2}$ appearing in the denominator of $\Delta_{8}^{(2)}$, and can be of a factor of a few. In the limit of $m_{\nu_{1}} \ll m_{\nu_{2,3}}$ we have:

$$
\frac{B_{\mu \rightarrow e \gamma}}{B_{\tau \rightarrow \mu \gamma}} \sim \frac{c_{12}^{2} c_{13}^{2}}{\left(c_{12} c_{23} s_{13} \mp s_{12} s_{23}\right)^{2}} \approx 7.3(3.2),
$$



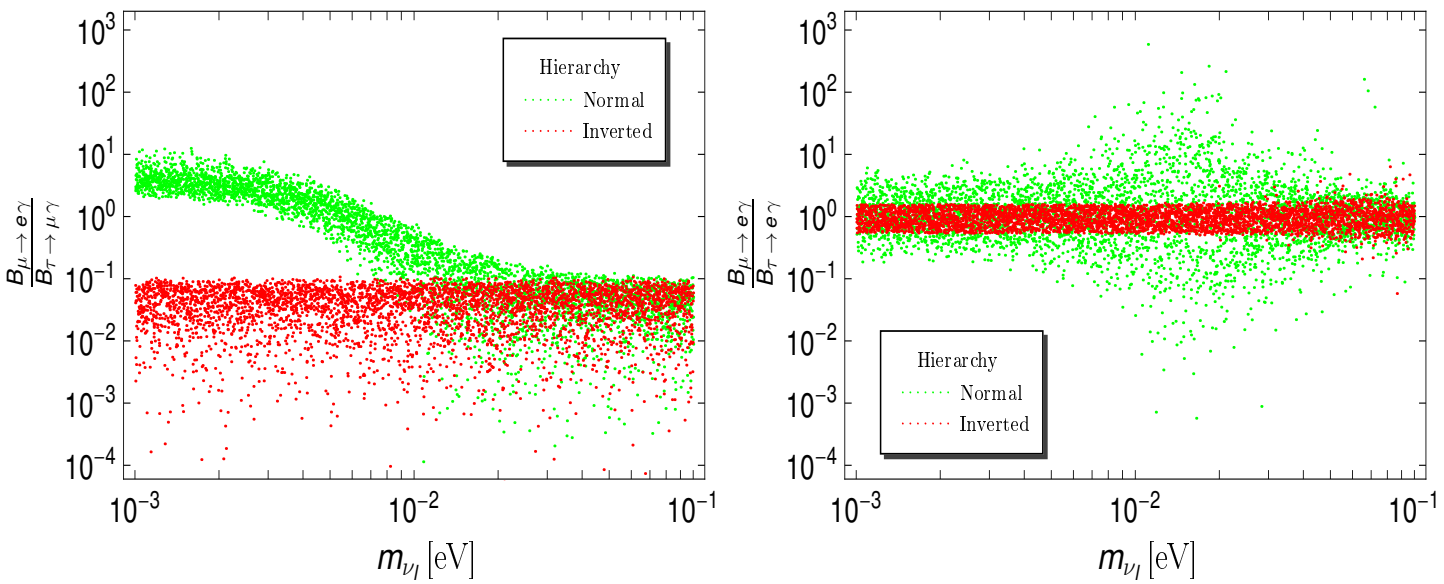

Figure 1. Scatter plots of the ratios $\frac{B_{\mu \rightarrow e \gamma}}{B_{\tau \rightarrow \mu \gamma}}$ (left) and $\frac{B_{\mu \rightarrow e \gamma}}{B_{\tau \rightarrow e \gamma}}$ (right) as a function of the mass of the lightest neutrino. Green points correspond to NH with $m_{\nu_{l}}=m_{\nu_{1}}$. Red points correspond to IH with $m_{\nu_{l}}=m_{\nu_{3}}$. The density of points depends on arbitrary details of the sampling procedure and does not represent the likelihood of different regions.

where $c_{i j} \equiv \cos \theta_{i j}$ and $s_{i j} \equiv \sin \theta_{i j}$ and the $-(+)$ sign in the denominator of the first equality corresponds to $\delta=0(\delta=\pi)$. The numerical estimate in the last equality is obtained using the best fit values of the mixing angles, again for $\delta=0(\delta=\pi)$. When $m_{\nu_{1}}^{2} \gg \Delta m_{\text {sol }}^{2}$ and $m_{\nu_{1}} \approx m_{\nu_{2}}$, the contributions to $\mu \rightarrow e \gamma$ proportional to $\theta_{12}$ suffer a strong GIM suppression, and the decay rate becomes proportional to $\theta_{13}^{2}$. This behavior is seen clearly in figure 1 (left) for values of $m_{\nu_{1}} \approx 10^{-2} \mathrm{eV}$.

For the IH, in the limit $m_{\nu_{3}} \ll m_{\nu_{1,2}}$ and independently of the value of $\delta$ we obtain:

$$
\frac{B_{\mu \rightarrow e \gamma}}{B_{\tau \rightarrow \mu \gamma}} \sim \frac{s_{13}^{2}}{c_{13}^{2} c_{23}^{2}} \approx 2 s_{13}^{2}
$$

Approximately the same result is obtained also in the limit of large masses $m_{\nu_{i}} \gg \sqrt{\Delta m_{\text {atm }}^{2}}$, which explains why for $m_{\nu_{1}} \rightarrow 10^{-1} \mathrm{eV}$ the results for $\mathrm{IH}$ and $\mathrm{NH}$ converge.

Results for the ratio of the $\mu$ and $\tau$ radiative decays into electrons are depicted in the right panel in figure 1. For NH, in the $m_{\nu_{1}} \ll m_{\nu_{2,3}}$ limit and neglecting terms suppressed by $\theta_{13}$ we obtain

$$
\frac{B_{\mu \rightarrow e \gamma}}{B_{\tau \rightarrow e \gamma}} \sim \cot _{23}^{2},
$$

while in the $\mathrm{IH}$ and quasi degenerate case of large masses

$$
\frac{B_{\mu \rightarrow e \gamma}}{B_{\tau \rightarrow e \gamma}} \sim \tan _{23}^{2}
$$

is obtained, approximately independently of the values of $\delta$ and $\theta_{13}$. A glance at the second panel in figure 1 confirms in fact that the $\mu / \tau$ ratio for decays into electrons remain centered around one for all values of $m_{\nu_{l}}$. For NH, $B_{\tau \rightarrow e \gamma}\left(B_{\mu \rightarrow e \gamma}\right)$ can vanish when $\delta=0(\pi)$ and $\frac{m_{\nu_{1}}^{2}+m_{\nu_{2}}^{2}}{2 m_{\nu_{3}}^{2}} \approx \frac{\Delta m_{\text {sol }}^{2}}{\Delta m_{\text {atm }}^{2}} \frac{s_{12} c_{12}}{s_{13}}$, which can be satisfied with $m_{\nu_{1}}$ in the few $\times 10^{-2} \mathrm{eV}$ range. This 

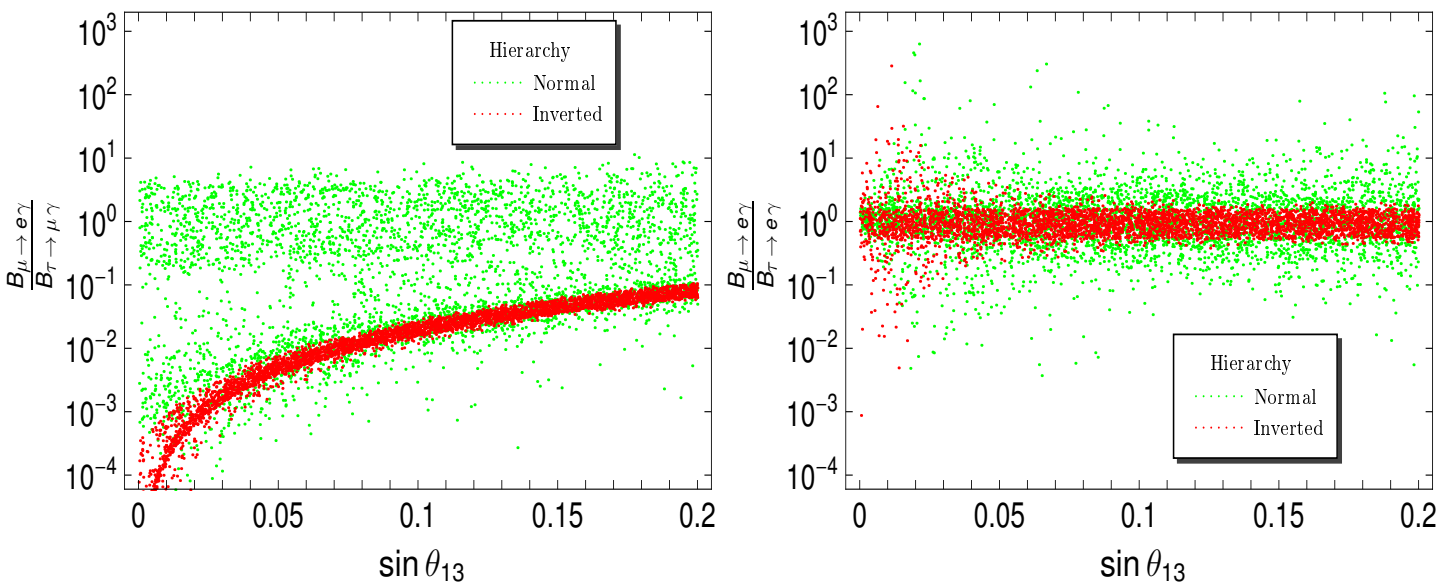

Figure 2. Same than figure 1 but as a function of $\sin \theta_{13}$.
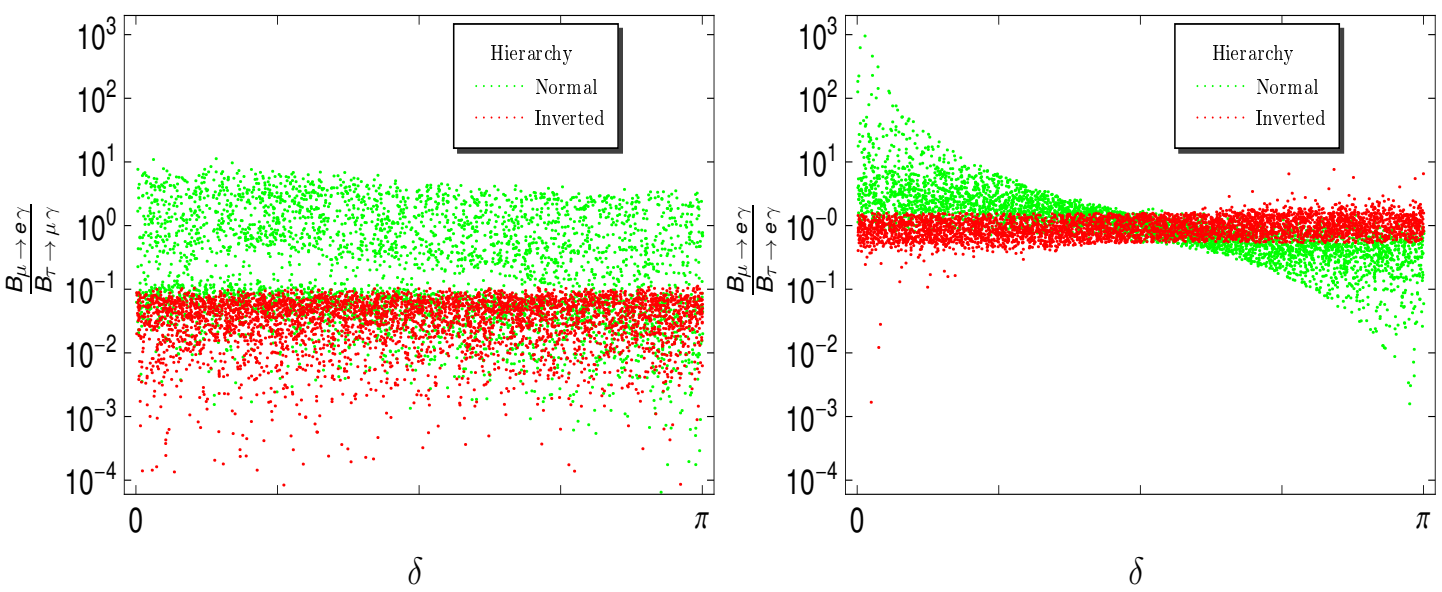

Figure 3. Same than figure 1 but as a function of the Dirac phase $\delta$.

accidental enhancement (suppression) of the ratio can be clearly seen in the central region of the second scatter plot.

For completeness, we have also studied the dependence of these two ratios as a function of $\sin \theta_{13}$ and of the Dirac phase $\delta$. The first panel in figure 2 confirms that for $\mathrm{NH}$ a factor of a few enhancement of $B_{\mu \rightarrow e \gamma}$ with respect to $B_{\tau \rightarrow \mu \gamma}$ is possible, independently of the value of $\theta_{13}$. For IH instead, $B_{\mu \rightarrow e \gamma}$ is suppressed as $\theta_{13}^{2}$, in agreement with eq. (2.24).

Figure 3 depicts the correlations of the results with the Dirac phase $\delta$. For NH the enhancements of $B_{\mu \rightarrow e \gamma}$ can occur for all values of the phase. In contrast, $B_{\mu \rightarrow e \gamma}$ can dominate over $B_{\tau \rightarrow e \gamma}$ only if $\delta<\frac{\pi}{2}$. Note that the extremely large enhancements (suppressions) that can be seen in the second panel of this figure for $\delta \rightarrow 0(\delta \rightarrow \pi)$ are not parametric effects, but arise from the already mentioned accidental cancellations that can occur when $m_{\nu_{1}} \sim 10^{-2} \mathrm{eV}$.

In summary, the new MLFV scenario that we have been discussing is characterized by a quite different phenomenology from the case previously studied in [6] since, in contrast to 
that case, it allows the branching fraction $B_{\mu \rightarrow e \gamma}$ to dominate over $B_{\tau \rightarrow \mu \gamma}$ and $B_{\tau \rightarrow e \gamma}$. The enhancement with respect $B_{\tau \rightarrow \mu \gamma}$ that occurs in the NH case does not exceed a factor of a few, but it is parametric in the small values of $m_{\nu_{1}}$. The strong enhancement with respect to $B_{\tau \rightarrow e \gamma}$ instead is due to accidental cancellations that suppress this process, and that become particularly efficient when $\delta$ is close to zero.

Needless to say, since the ratio of normalized branching ratios of other LFV processes like for example $B_{\mu \rightarrow 3 e}, B_{\tau \rightarrow 3 \mu}, B_{\tau \rightarrow 3 e}$ are controlled by the same LFV factors $\Delta_{8}^{(2)}$, they are characterized by a completely similar pattern of enhancements/suppressions.

In view of the ongoing high sensitivity experimental searches for LFV processes [26-28] besides comparing the rates for different LFV channels it is also of primary interest to give an estimate of the absolute values of the branching fractions. In the most favorable case, in which $\Delta_{8}^{(2)}$ is a matrix with $\mathcal{O}(1)$ entries, it is easy to derive a rough estimate:

$$
B_{\mu \rightarrow e \gamma} \approx 1536 \pi^{3} \alpha \frac{v^{4}}{\Lambda^{4}}
$$

Comparing eq. (2.27) with the present experimental limit $B_{\mu \rightarrow e \gamma}^{\exp } \lesssim 10^{-11}[29,30]$ we can conclude that the scale of LFV should be rather large: $\Lambda \gtrsim 400 \mathrm{TeV}$.

\section{Conclusions}

In this paper we have studied MLFV extensions of the type-I seesaw. This model is characterized by the group of broken symmetries $\mathrm{SU}(3)_{\ell} \times \mathrm{SU}(3)_{N} \times \mathrm{SU}(3)_{e} \times \mathrm{U}(1)_{L} \times \mathrm{U}(1)_{R}$, where the last Abelian factor $\mathrm{U}(1)_{R}$ can be identified with phase rotations of the $\mathrm{RH}$ leptons $e$ or of the RH neutrinos $N$, being this second choice phenomenologically more interesting and thus the one that we have adopted. We have parametrized the breaking of the Abelian symmetries by means of two spurions $\mu_{L}$ and $\epsilon_{\nu}$, and the breaking of the semi-simple flavour group $\mathrm{SU}(3)^{3}$ by means of three 'non-Abelian' spurions $Y_{e}, Y_{\nu}$ and $Y_{M}$, being the first two related to the charged leptons and RH neutrinos Yukawa couplings and the last one with the $\mathrm{RH}$ neutrinos mass matrix.

We have seen that formulating a predictive MLFV framework for the type I seesaw model with three RH neutrinos, that is a framework in which LFV effects can be completely described in terms of low energy observables, is possible only if the number of relevant LFV free parameters of the seesaw is reduced. This can be achieved by imposing specific conditions on the structure of the non-Abelian spurions. We have identified two basic possibilities that can be elegantly realized by assuming from the beginning that the underlying symmetry of the type-I seesaw is a subgroup of the full flavour symmetry. These two possibilities are:

A. $Y_{\nu}$ is proportional to a unitary matrix. This means that there is a basis in which $Y_{\nu} \sim I_{3 \times 3}$ being $I_{3 \times 3}$ the identity in flavour space. This scenario can be realized by restricting the flavour symmetry $\mathrm{SU}(3)_{e} \times \mathrm{SU}(3)_{N}$ to its subgroup $\mathrm{SU}(3)_{\ell+N}$.

B. $Y_{M} \sim I_{3 \times 3}$ and $Y_{\nu}$ is a matrix with real entries $Y_{\nu}=Y_{\nu}^{*}$. The symmetry reduction that realizes this second possibility is $\mathrm{SU}(3)_{N} \rightarrow O(3)_{N}$. In this case CP conservation in the lepton sector must be also imposed to ensure the reality of $Y_{\nu}$. 
While the second possibility is well studied [6, 7], the first one is new, and yields a quite different phenomenology from case $\mathbf{B}$. Most remarkably, it allows for sizable enhancements of processes involving the $\mu \rightarrow e$ transition with respect to LFV processes involving the $\tau$ lepton.

As regards the two broken Abelian factors $\mathrm{U}(1)_{L}$ and $\mathrm{U}(1)_{N}$, we have found that by parametrizing their breaking independently from the breaking of the semi-simple groups of flavour transformations leaves open unexpected possibilities: (i) the mass scale of the $\mathrm{RH}$ neutrinos gets decoupled from the large seesaw scale $\mu_{L}$, since the former breaks $\mathrm{U}(1)_{N}$ by two units while, in the low energy effective theory, the latter only breaks $\mathrm{U}(1)_{L}$. If $\mathrm{U}(1)_{N}$ breaking is small, say $\epsilon_{\nu}<10^{-5}$, then the RH neutrinos can be at the $\mathrm{TeV}$ scale or even below. (ii) Higher dimension operators, like $(\bar{N} N)(\bar{q} q)$, that could be effective for producing the $\mathrm{RH}$ neutrinos, do not break $\mathrm{U}(1)_{N}$, and therefore are not affected by any strong suppression of the RH neutrino masses. Of course, in our framework, such an intriguing scenario represents just an open possibility that is allowed by the symmetries of the type-I seesaw. It remains to be seen if explicit models realizing this scenario can be constructed.

\section{Acknowledgments}

R. A. acknowledges CICYT through the project FPA2009-09017, CAM through the project HEPHACOS, P-ESP-00346, and financial support from the MICINN grant BES-2010037869. G. I. acknowledges the support of the Technische Universität München - Institute for Advanced Study, funded by the German Excellence Initiative. L. M. acknowledges the German 'Bundesministerium für Bildung und Forschung' under contract 05H09WOE. R. A. and L. M. thank the theoretical group of the Laboratori Nazionale di Frascati for hospitality during the development of this project.

\section{References}

[1] R.S. Chivukula and H. Georgi, Composite Technicolor Standard Model, Phys. Lett. B 188 (1987) 99 [SPIRES].

[2] L.J. Hall and L. Randall, Weak scale effective supersymmetry, Phys. Rev. Lett. 65 (1990) 2939 [SPIRES].

[3] A.J. Buras, P. Gambino, M. Gorbahn, S. Jager and L. Silvestrini, Universal unitarity triangle and physics beyond the standard model, Phys. Lett. B 500 (2001) 161 [hep-ph/0007085] [SPIRES].

[4] G. D'Ambrosio, G.F. Giudice, G. Isidori and A. Strumia, Minimal flavour violation: An effective field theory approach, Nucl. Phys. B 645 (2002) 155 [hep-ph/0207036] [SPIRES].

[5] S. Weinberg, Baryon and Lepton Nonconserving Processes, Phys. Rev. Lett. 43 (1979) 1566 [SPIRES].

[6] V. Cirigliano, B. Grinstein, G. Isidori and M.B. Wise, Minimal flavor violation in the lepton sector, Nucl. Phys. B 728 (2005) 121 [hep-ph/0507001] [SPIRES].

[7] V. Cirigliano and B. Grinstein, Phenomenology of minimal lepton flavor violation, Nucl. Phys. B 752 (2006) 18 [hep-ph/0601111] [SPIRES]. 
[8] V. Cirigliano, G. Isidori and V. Porretti, CP violation and Leptogenesis in models with Minimal Lepton Flavour Violation, Nucl. Phys. B 763 (2007) 228 [hep-ph/0607068] [SPIRES].

[9] G.C. Branco, A.J. Buras, S. Jager, S. Uhlig and A. Weiler, Another look at minimal lepton flavour violation, $\ell_{i} \rightarrow \ell_{j} \gamma$, leptogenesis, and the ratio $M_{\nu} / \Lambda_{\mathrm{LFV}}$, JHEP 09 (2007) 004 [hep-ph/0609067] [SPIRES].

[10] S. Davidson and F. Palorini, Various definitions of minimal flavour violation for leptons, Phys. Lett. B 642 (2006) 72 [hep-ph/0607329] [SPIRES].

[11] M.B. Gavela, T. Hambye, D. Hernandez and P. Hernández, Minimal Flavour Seesaw Models, JHEP 09 (2009) 038 [arXiv:0906.1461] [SPIRES].

[12] A.S. Joshipura, K.M. Patel and S.K. Vempati, Type I seesaw mechanism for quasi degenerate neutrinos, Phys. Lett. B 690 (2010) 289 [arXiv:0911.5618] [SPIRES].

[13] P. Minkowski, $\mu \rightarrow$ er at a Rate of One Out of 1-Billion Muon Decays?, Phys. Lett. B 67 (1977) 421 [SPIRES].

[14] M. Gell-Mann, P. Ramond and R. Slansky, Proceedings of the Supergravity Stony Brook Workshop, New York U.S.A. (1979), P. Van Nieuwenhuizen and D. Freedman eds., North-Holland, Amsterdam The Netherlands (1980).

[15] T. Yanagida, Proceedings of the Workshop on Unified Theories and Baryon Number in the Universe, Tsukuba Japan (1979), A. Sawada and A. Sugamoto eds., KEK Report No. 79-18, Tsukuba Japan (1979).

[16] R.N. Mohapatra and G. Senjanović, Neutrino mass and spontaneous parity nonconservation, Phys. Rev. Lett. 44 (1980) 912 [SPIRES].

[17] J. Kersten and A.Y. Smirnov, Right-Handed Neutrinos at LHC and the Mechanism of Neutrino Mass Generation, Phys. Rev. D 76 (2007) 073005 [arXiv:0705.3221] [SPIRES].

[18] E. Bertuzzo, P. Di Bari, F. Feruglio and E. Nardi, Flavor symmetries, leptogenesis and the absolute neutrino mass scale, JHEP 11 (2009) 036 [arXiv:0908.0161] [SPIRES].

[19] P.F. Harrison, D.H. Perkins and W.G. Scott, Tri-bimaximal mixing and the neutrino oscillation data, Phys. Lett. B 530 (2002) 167 [hep-ph/0202074] [SPIRES].

[20] Z.-z. Xing, Nearly tri-bimaximal neutrino mixing and CP-violation, Phys. Lett. B 533 (2002) 85 [hep-ph/0204049] [SPIRES].

[21] D. Aristizabal Sierra, F. Bazzocchi, I. de Medeiros Varzielas, L. Merlo and S. Morisi, Tri-Bimaximal Lepton Mixing and Leptogenesis, Nucl. Phys. B 827 (2010) 34 [arXiv: 0908.0907] [SPIRES].

[22] G.L. Fogli, E. Lisi, A. Marrone, A. Palazzo and A.M. Rotunno, Hints of $\theta_{1} 3>0$ from global neutrino data analysis, Phys. Rev. Lett. 101 (2008) 141801 [arXiv:0806.2649] [SPIRES].

[23] G.L. Fogli, E. Lisi, A. Marrone, A. Palazzo and A.M. Rotunno, What we (would like to) know about the neutrino mass, arXiv:0809.2936 [SPIRES].

[24] T. Schwetz, M.A. Tortola and J.W.F. Valle, Three-flavour neutrino oscillation update, New J. Phys. 10 (2008) 113011 [arXiv:0808. 2016] [SPIRES].

[25] M. Maltoni and T. Schwetz, Three-flavour neutrino oscillation update and comments on possible hints for a non-zero $\theta_{13}$, PoS (IDM2008) 072 [arXiv:0812.3161] [SPIRES]. 
[26] MEG collaboration, F. Cei, Lepton flavour violation experiments in LHC era, J. Phys. Conf. Ser. 259 (2010) 012010.

[27] MEG collaboration, S. Mihara, MEG experiment at the Paul Scherrer Institute, Nucl. Phys. A $\mathbf{8 4 4}$ (2010) 150c-154c [SPIRES].

[28] MEG collaboration, S. Ritt, Status of the $M E G$ expriment $\mu \rightarrow e \gamma$, Nucl. Phys. Proc. Suppl. 162 (2006) 279 [SPIRES].

[29] MEGA collaboration, M.L. Brooks et al., New Limit for the Family-Number Non-conserving Decay $\mu^{+} \rightarrow e_{\gamma}^{+}$, Phys. Rev. Lett. 83 (1999) 1521 [hep-ex/9905013] [SPIRES].

[30] MEGA collaboration, M. Ahmed et al., Search for the lepton-family-number nonconserving decay $\mu \rightarrow e^{+} \gamma$, Phys. Rev. D 65 (2002) 112002 [hep-ex/0111030] [SPIRES]. 